\title{
EVALUACIÓN DE LA PRODUCCIÓN DE METANO EN LA DIGESTIÓN ANAEROBIA DE VINAZAS PRETRATADAS CON OZONO
}

\author{
María Fernanda Durán Meneses ${ }^{1}$ \\ IRMA JANETH SANABRIA GÓMEZ ${ }^{2}$ \\ NELSON GUTIÉRREZ GUZMÁN ${ }^{3}$
}

\section{RESUMEN}

Este artículo presenta la evaluación de la remoción de compuestos fenólicos presentes en la vinaza, utilizando un proceso avanzado de oxidación (PAO) basado en ozono acoplado a un proceso biológico anaerobio. Las evaluaciones de los tratamientos se realizaron determinando los cambios en la concentración de compuestos fenólicos en función del tiempo de aplicación de ozono (7 - 15 - 30 minutos) y pH del sustrato $(4,42-7,47)$ con el fin de determinar la influencia de estos factores sobre la producción de metano en el proceso de digestión anaerobia. Los resultados indican que el pH neutro en la vinaza favorece un 27 \% más la remoción de compuestos fenólicos en comparación al pH ácido. Se observó que después del proceso de ozonización de vinazas a pH neutro la concentración de fenoles totales disminuye un 62 \% influenciando la producción de metano en un 70 \% con respecto a la producción de metano obtenida a partir de vinazas crudas.

PALABRAS CLAVE: PAO, ozonización, digestión anaerobia, producción de metano.

\section{EVALUATION OF METHANE PRODUCTION IN ANAEROBIC DIGESTION OF VINASSE PRETREATED WITH OZONE}

\section{ABSTRACT}

This paper presents the results of the methane production from anaerobic digestion of vinasse derived from bioethanol manufacturing, using an advanced oxidation process (AOP) with pre-treatment based on ozone. Evaluations of the treatments were performed by determining the observed changes on the concentrations the phenolic compounds on the methane production in the anaerobic digestion process can be a function of the ozone application time (7 - 15 - 30 minutes) and $\mathrm{pH}(4,2-7,47)$. The neutral $\mathrm{pH}$ favours by $27 \%$ the removal of phenolic compounds in the vinasse in comparison to acid $\mathrm{pH}$. It was also observed that after of vinasse ozonation process at neutral $\mathrm{pH}$, the concentration of phenol decreased by $62 \%$ influencing the methane production by $70 \%$ with respect to the production obtained from raw vinasse.

KEYWORDS: AOP, ozonation, anaerobic digestion, methane production.

1 Ingeniera agrícola, Universidad Surcolombiana. Neiva, Colombia. Magíster en Microbiología Agrícola, Universidade Federal de Viçosa, Brasil.

2 Licenciada en Biología, Universidad Pedagógica Nacional. PhD. Microbiología, Instituto de Microbilogía y Virología. Postdoctorado Ecole Polytechnique Federale De Lausanne, Suiza. Docente Universidad del Valle. Cali, Colombia

3 Ingeniero agrícola, Universidad Surcolombiana, Neiva, Colombia. Magíster en Ingeniería de Alimentos, Universidad Politécnica de Valencia, España. PhD. y Postdoctorado en Tecnología de Alimentos, Universidad Politécnica de Valencia, España. Docente Universidad Surcolombiana, Neiva, Colombia

Autor de correspondencia: Durán, M.F. (María Fernanda). Universidad Surcolombiana. Av. Pastrana Borrero, carrera 1‥ Neiva, Huila, Colombia,/ Tel.: (57) 8754753. Correo electrónico: fernandaduram@gmail.com

\section{Historia del artículo:}

Artículo recibido: 13-VI-2015 / Aprobado:

Disponible online: 30 de enero de 2016

Discusión abierta hasta noviembre de 2016 


\section{AVALIAÇÃO DA PRODUÇÃO DE METANO NA DIGESTÃO ANAERÓBIA DA VINHAÇA PRÉ-TRATADA COM OZÔNIO}

\section{RESUMO}

Este trabalho apresenta a avaliação da remoção dos compostos fenólicos presentes na vinhaça, usando um processo de oxidação avançada (PAO), com base no ozono acoplado a um processo biológico anaeróbio. As avaliações dos tratamentos foram realizados por determinação das alterações na concentração de compostos fenólicos em função do tempo de aplicação de ozono (7 - 15 - 30 minutos) e pH do substrato (4,42 a 7,47), a fim de determinar a influência desses fatores sobre a produção de metano no processo de digestão anaeróbia. Os resultados indicam que o pH neutro favorece um 27\% de remoção de compostos fenólicos em comparação com pH ácido. Observou-se que após do processo de ozonização de vinhaças no pH neutro a concentração total de fenol diminui um $62 \%$ influenciando a produção de metano em $70 \%$ em relação à produção de metano obtido a partir de vinhaça cru.

PALAVRAS-CHAVE: PAO, ozonização, digestão anaeróbia, produção de metano.

\section{INTRODUCCIÓN}

El afán de reemplazar los combustibles fósiles por biocombustibles ha generado un crecimiento desmesurado en la producción de alcohol carburante (etanol), biodiesel y, consecuentemente, de los residuos producidos en cada proceso (Gil, 2011). En el caso particular de producción de etanol, se estimó que en el año 2014 se produjeron 90380 millones de litros de este alcohol en el mundo (GRFA, 2014), de los cuales Colombia, décimo productor mundial de etanol de caña de azúcar, produjo cerca de 450 millones de litros (Federación Nacional de Biocombustibles de Colombia, 2015). La vinaza es un residuo líquido resultante de la producción de etanol ya sea por destilación de la melaza fermentada o de la fermentación directa de los jugos de la caña de azúcar (García et al, 2008) y por cada litro de etanol producido se obtienen entre 0,8 y 3,0 litros de vinaza (Asocaña, 2011).

En Colombia los ingenios azucareros del valle geográfico del río Cauca, productores de etanol, han adoptado un manejo riguroso de sus residuos siendo fertilizante el uso más amplio dado a las vinazas, aunque otras alternativas para disposición final de este residuo han surgido como respuesta al aumento de salinidad en los suelos fertilizados con vinazas, entre las cuales se destacan la incineración, la digestión anaerobia, la deshidratación y el compostaje (Cenicaña, 2010); sin embargo, es importante contemplar medidas adicionales para su utilización debido a que ninguna de estas alternativas por si sola es viable para manejar todo el volumen de vinaza producido. Dávila et al., (2009) evaluaron el proceso de electro-flotación/ oxidación de vinazas, consiguiendo reducciones en demanda química de oxígeno en un $58 \%$ así como Yusuf (2007) quien logró una reducción del 90 \% en carbono orgánico total en vinazas por medio de electrocoagulación con el uso de un electrolito soporte y la adición gradual de peróxido de hidrógeno y, en 2012 España-Gamboa et al., trataron con digestión anaerobia vinazas provenientes de la destilación de etanol alcanzando una reducción de $69 \%$ en la demanda química de oxígeno y 0,263 $\mathrm{m}^{3} \mathrm{~kg} \mathrm{DQO}_{\text {adicionada }}$ de producción de metano, el cual es considerado una promisoria fuente de energía renovable.

En este sentido, en un sistema biológico de digestión anaerobia el contenido orgánico de la vinaza, 156 g DQO.L $L^{-1}$ (Caicedo, 2010) puede ser aprovechado y transformado en metano; sin embargo, los 
compuestos tóxicos presentes en la vinaza, principalmente fenoles y polifenoles (Robles-González et al., 2012) afectan el desarrollo del sistema biológico y hacen imprescindible el uso de un tratamiento previo al biológico, que permita la degradación de compuestos inhibidores para el aprovechamiento integral de la vinaza.

Los Procesos Avanzados de Oxidación (PAO) han sido usados como alternativa de descontaminación de agua, siendo capaces de mineralizar por completo los contaminantes orgánicos (Malato et al. 2009) y como pre-tratamiento al proceso de hidrólisis enzimática en sistemas de fermentación con el objetivo de incrementar la digestibilidad de materiales lignocelulósicos (Travaini et al., 2013). No obstante, estas tecnologías necesitan mayor desarrollo para que sean económicamente viables. De esta manera, los acoples con sistemas biológicos representan una de las mejores opciones para disminuir los costos de implementación y los impactos ambientales producidos por los mismos (GarcíaMontaño et al., 2008).

Este estudio tuvo por objeto evaluar el ozono como proceso avanzado de oxidación para degradar compuestos fenólicos presentes en la vinaza generada a partir de la producción de etanol de caña de azúcar, acoplado al proceso de digestión anaerobia para la obtención de metano como biocombustible de segunda generación.

\section{MATERIALES Y MÉTODOS}

\subsection{Localización}

Todos los ensayos se realizaron en el Laboratorio de Microbiología Ambiental, del Programa de Ingeniería Sanitaria y Ambiental de la ciudadela universitaria Meléndez, de la Universidad del Valle, en Santiago de Cali - Colombia.

\subsection{Procedencia de la vinaza y el inócullo}

Las vinazas fueron donadas por el ingenio Mayagüez, ubicado en el municipio de Candelaria

- Valle del Cauca, provenientes del proceso de destilación de caña de azúcar en la producción de alcohol carburante; el inóculo utilizado en el proceso de digestión anaerobia fue tomado del reactor UASB de la central de sacrifico animal de Cavasa, ubicado en el municipio de Candelaria - Valle del Cauca.

\subsection{Caracterización de las vinazas}

Antes y después del tratamiento con ozono la vinaza fue caracterizada mediante los parámetros de Demanda Química de Oxígeno (DQO), pH, Sólidos Suspendidos Totales (SST), Demanda Bioquímica de Oxígeno $\left(\mathrm{DBO}_{5}\right)$, nitrógeno amoniacal $\left(\mathrm{N}-\mathrm{NH}_{3}\right)$, sulfuro $\left(\mathrm{S}^{-2}\right)$ y fenoles totales según el Standard Methods (APHA, 2005).

\subsection{Remoción de compuestos fenólicos mediante PAO}

Las vinazas fueron pre-tratadas mediante un proceso avanzado de oxidación (PAO): Ozonólisis, utilizando una columna de vidrio $40 \mathrm{~cm}$ de alto y 5 $\mathrm{cm}$ de diámetro, la cual opera con un flujo de 0,5 l/ min de mezcla oxígeno/ozono bajo condiciones de temperatura ambientales $\left(26^{\circ} \mathrm{C}\right)$; la concentración de ozono empleada fue de $50 \mathrm{mg} \mathrm{O}^{3} \mathrm{~L}^{-1}$. La vinaza se dispuso cruda (55 \% de SST) en la columna de vidrio. Con el objetivo de evaluar el sistema de burbujeo y garantizar el flujo de ozono a través de la muestra, se recirculó la espuma generada durante el proceso, de esta manera se logró un contacto constante entre la vinaza y el flujo de ozono (Figura 1).

El difusor empleado es de vidrio poroso de burbuja fina en la que la eficiencia de transferencia de ozono se maximiza aumentando el área interfacial (Gogate y Pandit, 2004).

Se utilizó una concentración de ozono de $50 \mathrm{mg}$ $\mathrm{L}^{-1}$, atendiendo lo recomendado por Caicedo (2010), por ser esta la concentración considerada más eficiente para la eliminación de compuestos fenólicos en vinazas; el flujo de oxigeno/ozono utilizado fue de $0,5 \mathrm{l} / \mathrm{min}$. Teniendo en cuenta que el objetivo de este proyecto fue la remoción de los compuestos fenólicos en vinazas causando el menor cambio en 


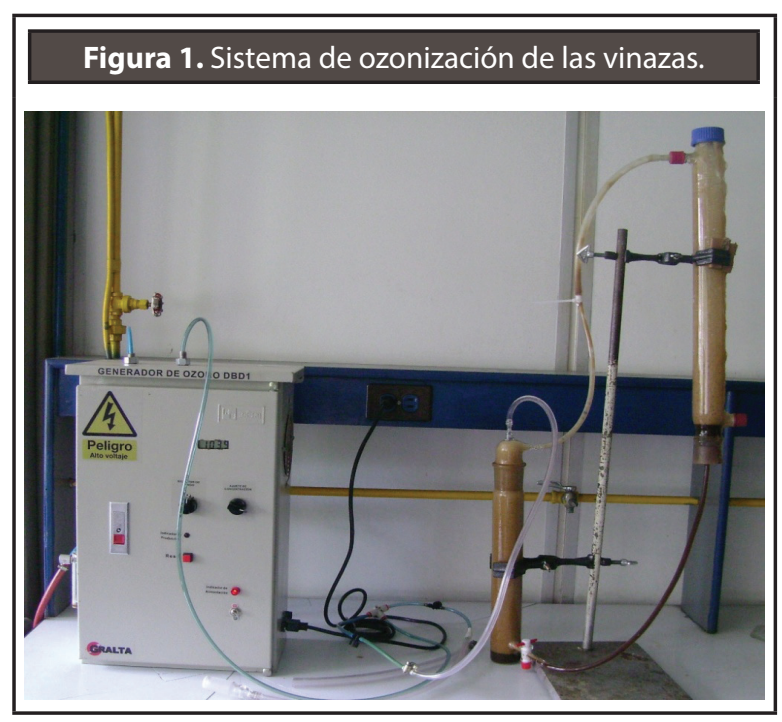

\begin{tabular}{|c|c|c|}
\hline \multicolumn{3}{|c|}{$\begin{array}{c}\text { Tabla 1. Condiciones de operación } \\
\text { para el proceso de ozonólisis* }\end{array}$} \\
\hline Pre-tratamiento & $\begin{array}{c}\text { Tiempo de aplicación } \\
\text { (min) }\end{array}$ & $p H$ \\
\hline 1 & 7 & 7,47 \\
\hline 2 & 7 & 4,42 \\
\hline 3 & 15 & 7,47 \\
\hline 4 & 15 & 4,42 \\
\hline 5 & 30 & 7,47 \\
\hline 6 & 30 & 4,42 \\
\hline${ }^{*}$ El flujo de oxígeno/ & $\begin{array}{l}\text { zono utilizado fue de } 0,5 \mathrm{l} / \\
\text { ndiciones STD }\end{array}$ & in en \\
\hline
\end{tabular}

su composición orgánica para ser aprovechada en la digestión anaerobia, se expuso el sustrato al ozono con periodos cortos de aplicación, evaluando el pretratamiento a pH 7,47 y 4,42 (Tabla 1).

\subsection{Montaje del ensayo de digestión anaerobia}

El ensayo de digestión anaerobia fue realizado en reactores de $120 \mathrm{ml}$, los cuales llevan tapa rosca y septo de caucho ajustado con sello metálico de 20 cm de diámetro, la siembra fue realizada en cámara de anaerobiosis. La fase líquida (volumen útil) estuvo compuesta por $33,5 \mathrm{ml}$ de agua destilada, 1,52

\begin{tabular}{|c|c|}
\hline \multicolumn{2}{|c|}{$\begin{array}{c}\text { Tabla 2. Tratamientos del ensayo } \\
\text { de digestión anaerobia }\end{array}$} \\
\hline \multicolumn{2}{|c|}{ Sustrato } \\
\hline Tratamiento & Vin Oz 7' $(\mathrm{pH} \mathrm{7,4)}$ \\
\hline 1 & Vin Oz 7' $(\mathrm{pH} \mathrm{4,4)}$ \\
\hline 2 & Vin Oz 15' $(\mathrm{pH} \mathrm{7,4)}$ \\
\hline 3 & Vin Oz 15' $(\mathrm{pH} \mathrm{4,4)}$ \\
\hline 4 & Vin Oz 30' $(\mathrm{pH} \mathrm{7,4)}$ \\
\hline 5 & Vin Oz 30' $(\mathrm{pH} \mathrm{4,4)}$ \\
\hline 6 & inóculo \\
\hline Control 1 & AGV \\
\hline Control 2 & Vinaza cruda \\
\hline Control 3 &
\end{tabular}

ml del inóculo (1,5 g SSV. L $\left.\mathrm{L}^{-1}\right), 0,4 \mathrm{ml}$ de solución de vitaminas y entre $0,56-0,84 \mathrm{ml}$ del sustrato debido a que las vinazas presentaron valores diferentes de DQO después de cada tratamiento con ozono, no obstante se garantizó la misma carga ( 4 g DQO. $\mathrm{L}^{-1}$ ) en todos los reactores mediante balances de masa por volumen. El balance de nutrientes realizado a la vinaza (ver sección 3.1 resultados) demostró que cumple con la relación carbono/nitrógeno requerida por los microorganismos anaerobios correspondiente a C/N = 20-30 (Lorenzo y Obaya, 2005, 2005) no siendo necesaria la adición de nutrientes en los reactores anaerobios.

Se realizaron 6 ensayos de digestión anaerobia con 3 réplicas cada uno y 3 réplicas para cada control $\left(\mathrm{C}_{1}=\right.$ inóculo, $\mathrm{C}_{2}=$ ácidos grasos volátiles (AGV) (73:23:4) y $\mathrm{C}_{3}=$ vinaza no tratada con ozono o vinaza cruda), como se indica en la Tabla 2. Las réplicas se mantuvieron durante los 25 días de experimentación. Los reactores fueron inoculados en anaerobiosis e incubados durante el ensayo a $35^{\circ} \mathrm{C}$ en posición invertida, de esta manera se generó un sello hidráulico hacia el tapón de caucho (Figura 2).

Vin $\mathrm{Oz}$ 7': vinaza ozonizada durante 7 minutos; Vin Oz 15': vinaza ozonizada durante 15 minutos; Vin Oz 30': vinaza ozonizada durante 30 minutos. AGV ácidos grasos volátiles. 


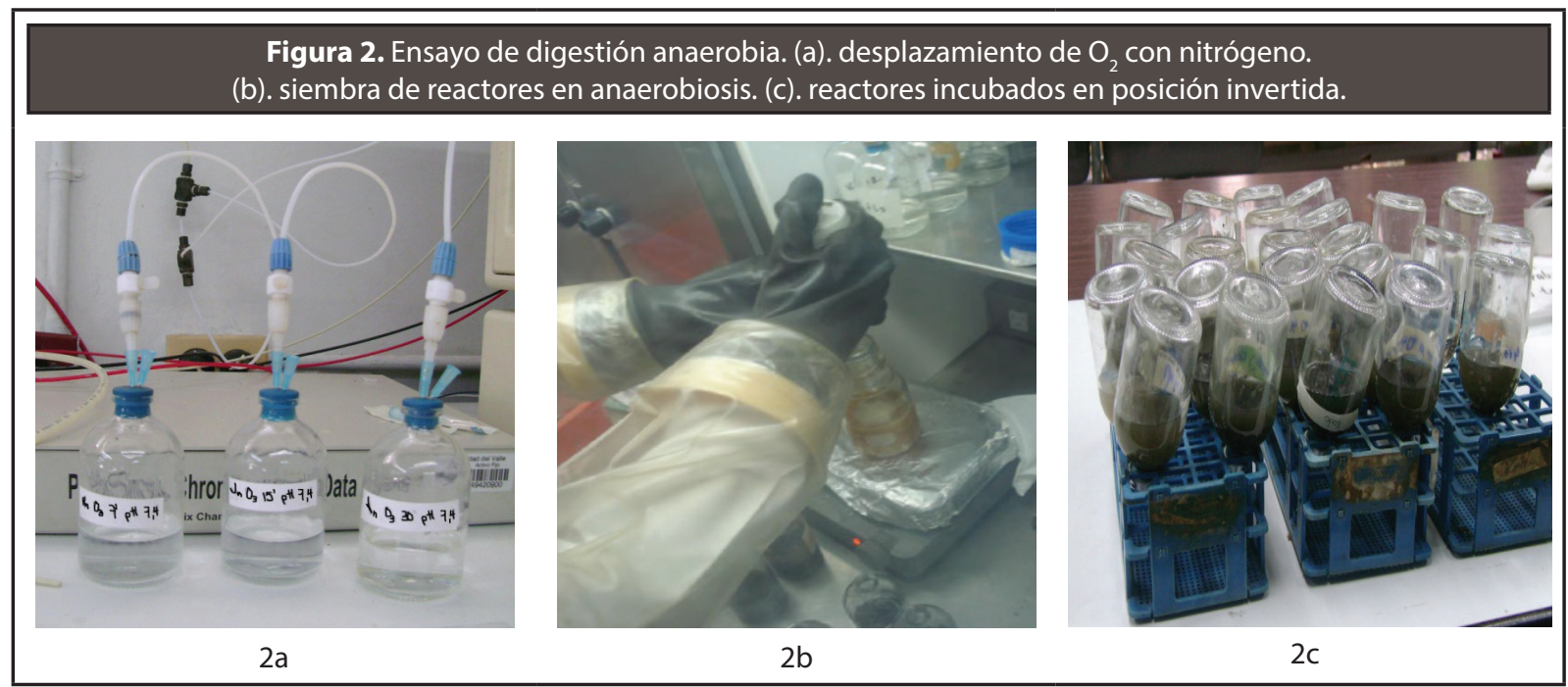

\subsection{Seguimiento de la producción de}

\section{metano}

Cada 3 días fue llevada a cabo la medición del metano producido mediante cromatografía gaseosa. El cromatógrafo de gases utilizado (Shimadzu GC 14, FID) está provisto de una columna Carbowax $20 \mathrm{M}(3 \%), \mathrm{H}_{3} \mathrm{PO}_{4}(1 \%)$ y supelco $80 / 100$. Los picos de metano se detectaron alrededor de 0,5 segundos, mediante el software Peak Simple 3.0. Las condiciones de operación del cromatógrafo fueron: $\mathrm{H}_{2}=40$ psi, Aire $=30$ psi, carrier $1=300$ psi y carrier $2=140$ psi. El gas de arrastre de ambos carriers fue nitrógeno molecular $\left(\mathrm{N}_{2}\right)$. La lectura de metano se realizó por duplicado, el volumen de inyección fue de $1 \mathrm{ml}$, se utilizó una jeringa con válvula para gases.

\subsection{Análisis estadístico}

Se realizaron pruebas de ANOVA de dos vías para 6 tratamientos, definiendo como factores el $\mathrm{pH}$ y el tiempo de ozonización y como variable respuesta la concentración de fenoles totales en $\mathrm{mg} \mathrm{L}^{-1}$ ácido gálico, se utilizó un nivel de Confianza del $95 \%$. Se realizaron también pruebas de ANOVA de dos vías para 6 tratamientos y como factores el $\mathrm{pH}$ y el tiempo de ozonización, como variable respuesta la producción de metano en ml, se utilizó un nivel de Confianza del $95 \%$. Se realizaron comprobaciones de normalidad, homocedasticidad e independencia de las observaciones para todas las pruebas, la determinación de grupos homogéneos se realizó mediante la prueba de HSD Tukey. Se utilizaron los paquetes estadísticos Minitab y StatGraphics Plus 5.1 para Windows (Manugistics, Inc., Rockville MD, USA).

\section{RESULTADOS Y DISCUSIÓN}

\subsection{Caracterización del sustrato}

En la Tabla 3 se presentan las características fisicoquímicas de la vinaza empleada en todos los ensayos de esta investigación proveniente del ingenio Mayagüez, Candelaria - Colombia.

El proceso de digestión anaerobia requiere una módica cantidad de nutrientes comparado con sistemas aerobios como consecuencia de la baja velocidad de crecimiento por parte de los microorganismos (Lorenzo y Obaya, 2005), así, los valores obtenidos de carbono (288787 mg L L $^{1}$ ), nitrógeno (888 $\mathrm{mg} \mathrm{L}^{-1}$ ) y fósforo (194 $\mathrm{mg} \mathrm{L}^{-1}$ ) en la vinaza evaluada aseguran el crecimiento de los microorganismos en los reactores biológicos de tratamiento anaerobio ya que el sustrato suple el ratio mínimo para el funcionamiento del sistema carbono/nitrógeno $=20-30$.

De acuerdo a esta caracterización la biodegradabilidad anaerobia de la vinaza es $\mathrm{DBO}_{5} / \mathrm{DQO}$ : 


\begin{tabular}{c|c|}
\hline \multicolumn{2}{|c|}{$\begin{array}{c}\text { Tabla 3. Caracterización fisicoquímica } \\
\text { de la vinaza empleada }\end{array}$} \\
\hline \multicolumn{2}{|c|}{ Valor* $^{*}$} \\
\hline Parámetro & 288787 \\
\hline DQO & 96030 \\
\hline $\mathrm{DBO}_{5}$ & 52000 \\
\hline $\mathrm{SST}$ & 4,42 \\
\hline $\mathrm{pH}$ & 888 \\
\hline Nitrógeno & 194 \\
\hline Fósforo & 182 \\
\hline $\mathrm{S}^{-2}$ & 180 \\
\hline N-NH & 306,120 \\
\hline Fenoles totales & $\mathrm{pH}$ son expresados en $\mathrm{mg} \mathrm{L}^{-1}$. \\
\hline *Todos los valores excepto
\end{tabular}

0,33; el resultado de esta relación significa que la diferencia entre el contenido de $\mathrm{DQO}$ y $\mathrm{DBO}_{5}$ es grande, indicando que existe una alta proporción de componentes no biodegradables. España-Gamboa y colaboradores (2011) reportan una alta concentración de fenoles en la vinaza proveniente de la industria vinícola de $474 \mathrm{mg} \mathrm{L}^{-1}$ lo cual resultó en inhibición del crecimiento de los microorganismos para las condiciones de digestión anaerobia. Las vinazas de caña de azúcar evaluadas en este estudio presentan una concentración de 306,120 $\mathrm{mg} \mathrm{L}^{-1}$, concentración considerada inhibitoria en el proceso anaerobio. Por otro lado, el nitrógeno amoniacal y el sulfuro a concentraciones entre $1700-14000 \mathrm{mg}$ $\mathrm{L}^{-1} \mathrm{y}$ entre 30 - $250 \mathrm{mg} \mathrm{L}^{-1}$ respectivamente, han sido reportados para ejercer efectos inhibitorios en el proceso de digestión anaerobia y consecuentemente en la producción de metano (Cirne et al., 2008; Parawira et al., 2008), sin embargo para el caso del nitrógeno amoniacal, concentraciones de $200 \mathrm{mg} \mathrm{L}^{-1}$ han sido reportadas como benéficas en el proceso de digestión anaerobia (Cirne et al., 2008). En este estudio, la concentración de nitrógeno amoniacal en la vinaza es de $180 \mathrm{mg} \mathrm{L}^{-1} \mathrm{y}$ de sulfuro es de $182 \mathrm{mg}$ $\mathrm{L}^{-1}$ siendo la concentración de nitrógeno amoniacal benéfica para el proceso biológico anaerobio, en contraste con la concentración de sulfuro.

\begin{tabular}{|c|c|c|c|}
\hline \multicolumn{4}{|c|}{ Tabla 4. Reducción de materia orgánica } \\
en los pre-tratamientos, en términos de DQO
\end{tabular}

\subsection{Reducción de compuestos orgánicos mediante PAO}

A partir de la vinaza cruda (ver Tabla 3) se realizó el pre-tratamiento con ozono a tiempos cortos de aplicación (7, 15 y 30 minutos), los valores de la concentración inicial, final y porcentajes de remoción tanto de DQO como de los compuestos fenólicos se observan en las Tablas 4 y $\mathbf{5}$, respectivamente.

El porcentaje de remoción de materia orgánica usando ozono como pre-tratamiento es más representativo en vinazas con pH ácido, alcanzando los valores más altos de remoción 25,49 - 33,53 para el pre-tratamiento aplicado a 15 y 30 minutos a $\mathrm{pH}$ 4,4 respectivamente. Las eficiencias de remoción de DQO a pH ácido están de acuerdo con lo reportado por Caicedo (2010) donde se concluye que el pH natural de la vinaza $(4,42)$ es el adecuado para aplicar una ozonización con fines de disminuir de la carga orgánica en este sustrato; sin embargo, a pesar de esta disminución la eficiencia obtenida al aplicar este pre-tratamiento es baja, de acuerdo a lo reportado por Martin Santos et al. (2003), la ozonización de vinazas no presenta altas tasas de remoción de materia orgánica, sino transformación de compuestos presentes en el residuo, por lo que es conveniente optar por esta tecnología para transformar compuestos de difícil degradación a compuestos más simples, sin disminuir en alto grado la concentración orgánica del residuo. 
Martín Santos et al, (2003) señalan que un pre-tratamiento con ozono en vinazas con $\mathrm{pH}$ natural $(4,42)$ es indicado para la remoción de compuestos fenólicos, en esa ocasión se evaluó la vinaza a un $\mathrm{pH}$ ácido $(4,5)$ y pH alcalino $(8,8)$ donde se evidencia que la reacción directa de oxidación (oxidación mediante el ozono molecular) es más eficiente para disminuir fenoles frente a la reacción indirecta (oxidación a través de los radicales hidroxilo); sin embargo, los resultados obtenidos en esta investigación (ver Tabla 5) indican que a pH cercanos a la neutralidad la disminución de compuestos fenólicos se da en mayor proporción que con pH ácidos, este hecho puede explicarse debido a que una condición de $\mathrm{pH}$ neutro favorece ambas reacciones de oxidación (directa e indirecta) concordando con Singer y Gurol (1983) quienes demuestran que la reacción de oxidación del fenol por ozono está influenciada por el $\mathrm{pH}$, incrementando la reacción a medida que aumenta el pH y con Jeworski y Heinzle (2000) quienes indican que la eficiencia del proceso de ozonización es mayor a pH neutros por la cantidad de ozono requerido para oxidar moléculas orgánicas. Además, Martín et al. (2005) exponen que el ozono es un oxidante selectivo para eliminar compuestos fenólicos en la vinaza, (reacción ozono-fenol entre 20 y 4 veces más rápida que con el resto de compuestos orgánicos presentes en el residuo) sin disminuir demasiado la carga orgánica transformable

Tabla 5. Remoción del contenido de fenoles totales en los pre-tratamientos.

\begin{tabular}{|c|c|c|c|}
\hline Pre-tratamiento & $\begin{array}{l}\text { Contenido } \\
\text { inicial de } \\
\text { fenol * }\end{array}$ & $\begin{array}{c}\text { Contenido } \\
\text { final de } \\
\text { fenol }^{*}\end{array}$ & $\begin{array}{c}\% \\
\text { remoción } \\
\text { de fenol }\end{array}$ \\
\hline 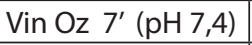 & 306,120 & 158,070 & 48,36 \\
\hline Vin Oz 15' $(\mathrm{pH} 7,4)$ & 306,120 & 134,200 & 56,16 \\
\hline Vin Oz $30^{\prime}(\mathrm{pH} 7,4)$ & 306,120 & 115,090 & 62,40 \\
\hline Vin Oz $7^{\prime}(\mathrm{pH} 4,4)$ & 306,120 & 243,180 & 20,56 \\
\hline Vin Oz 15' (pH 4,4) & 306,120 & 213,320 & 30,31 \\
\hline Vin Oz 30' $(\mathrm{pH} 4,4)$ & 306,120 & 196,770 & 35,72 \\
\hline
\end{tabular}

Tabla 6. ANOVA de dos vías: concentración de fenol frente al tiempo de ozonización y pH

\begin{tabular}{c|c|c|c}
\hline Factor & Suma de cuadrados & $\boldsymbol{F}$ & $\boldsymbol{P}$ \\
\hline $\begin{array}{c}\text { Tiempo de } \\
\text { Ozonización }\end{array}$ & 2877,2 & 39,89 & 0,000 \\
\hline $\mathrm{pH}$ & 22904,0 & 635,14 & 0,000 \\
\hline Tiempo-pH & 183,1 & 91,6 & 0,159 \\
\hline
\end{tabular}

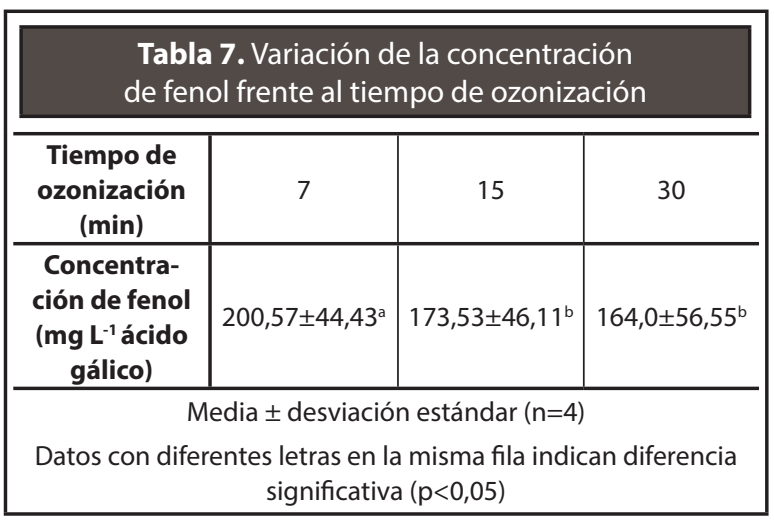

a metano. Siles et al. (2011) mencionan que la ozonización tiene una alta afinidad y selección por los compuestos fenólicos obteniendo subproductos de esta reacción que son fácilmente asimilables por las comunidades microbianas presentes en los procesos anaerobios, tales como aldehídos, cetonas, alcoholes y ácidos carboxílicos.

De acuerdo con el análisis de varianza mostrado en la Tabla 6, la disminución de la concentración de compuestos fenólicos en la vinaza no depende de la interacción entre el tiempo de aplicación de ozono y el $\mathrm{pH}$, depende por el contrario de la intensidad de los factores, es decir, los factores tiempo de aplicación de ozono y pH influyen individualmente sobre la disminución del fenol en el sustrato.

La Tabla 7 muestra la variación de la concentración de fenol para los diferentes tiempos de aplicación de ozono (7 - 15 - 30 minutos). En la tabla se observa que los compuestos fenólicos disminuyen a medida que el tiempo se hace más prolongado. El porcentaje de remoción de la concentración de compuestos fenólicos en la vinaza es de 34 \% en 


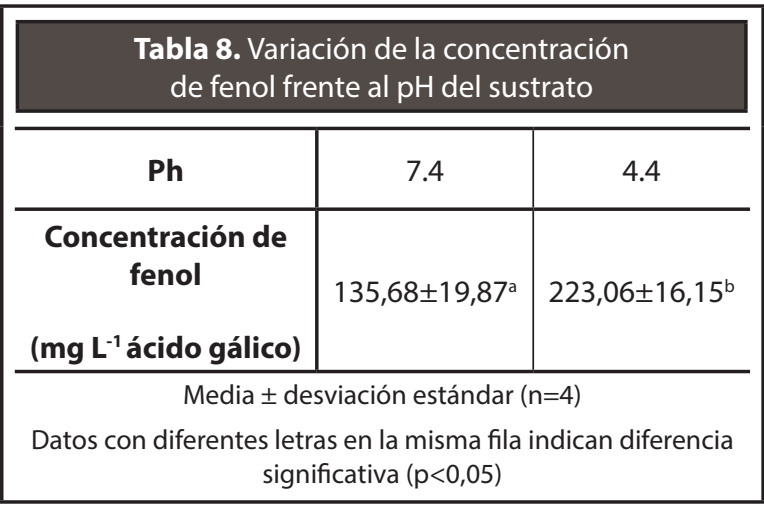

\begin{tabular}{|c|c|}
\hline \multicolumn{2}{|c|}{$\begin{array}{l}\text { Tabla 9. Resumen de la producción } \\
\text { de metano en los tratamientos. }\end{array}$} \\
\hline Sustrato & 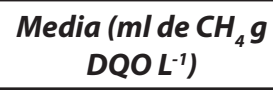 \\
\hline 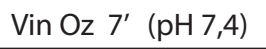 & $35,5 \pm 16,6$ \\
\hline Vin Oz $7^{\prime}(\mathrm{pH} 4,4)$ & $23,3 \pm 3,37$ \\
\hline 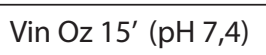 & $42,7 \pm 3,88$ \\
\hline Vin $\mathrm{Oz}_{15}(\mathrm{pH} 4,4)$ & $24,0 \pm 2,82$ \\
\hline Vin Oz $30^{\prime}(\mathrm{pH} 7,4)$ & $104,3 \pm 27,5$ \\
\hline Vin Oz 30' $(\mathrm{pH} 4,4)$ & $44,5 \pm 9,44$ \\
\hline Inóculo & $0,12 \pm 0,02$ \\
\hline Vinaza cruda & $28,7 \pm 19,7$ \\
\hline AGV & $114,3 \pm 8,4$ \\
\hline \multicolumn{2}{|c|}{ Media \pm desviación estándar $(n=3)$} \\
\hline
\end{tabular}

el minuto 7 y $43 \%$ y $46 \%$ en los minutos 15 y 30 , respectivamente.

De la misma manera, la Tabla 8 muestra la variación de la concentración del fenol a diferentes pH $(7,4-4,4)$. En la tabla se observa que los compuestos fenólicos presentan una mayor disminución a pH 7,4 lo que indica que la reacción de oxidación entre el ozono y el fenol aumenta cuando la vinaza presenta un $\mathrm{pH}$ cercano a la neutralidad con un porcentaje de remoción $62 \%$ y $35 \%$ para pH 7,4 y 4,4 respectivamente.

\subsection{Producción de metano}

En la Tabla 9 se presenta un resumen de la producción acumulada de metano de todas las pruebas de digestión anaerobia realizadas durante los

\begin{tabular}{|c|c|c|c|}
\hline \multicolumn{4}{|c|}{$\begin{array}{c}\text { Tabla 10. ANOVA de dos vías para producción de } \\
\text { metano en función del tiempo y } \mathrm{pH}\end{array}$} \\
\hline Factor & $\begin{array}{c}\text { Suma de } \\
\text { cuadrados }\end{array}$ & $\boldsymbol{F}$ & $\boldsymbol{P}$ \\
\hline $\begin{array}{c}\text { Tiempo de } \\
\text { ozonización }\end{array}$ & 11677 & 30,70 & 0,000 \\
\hline $\mathrm{pH}$ & 8991,67 & 47,28 & 0,000 \\
\hline Tiempo - pH & 535,48 & 1,41 & 0,2824 \\
\hline
\end{tabular}

\begin{tabular}{|c|c|c|c|}
\hline \multicolumn{3}{|c|}{ Tabla 11. Variación de la producción de metano } \\
frente al tiempo de ozonización \\
\hline $\begin{array}{c}\text { Tiempo de } \\
\text { ozonización } \\
\text { (min) }\end{array}$ & 7 & 15 & 30 \\
\hline $\begin{array}{c}\text { Producción } \\
\text { de metano } \\
\left.\text { (ml de } \mathbf{C H}_{4}\right)\end{array}$ & $18,33 \pm 21,54^{\text {a }}$ & $22,67 \pm 22,07^{\text {a }}$ & $74,4 \pm 37,5 \mathrm{~b}$ \\
\hline \multicolumn{4}{|c|}{ Media \pm desviación estándar ( $\mathrm{n}=6)$} \\
Datos con diferentes letras en la misma fila indican diferencia \\
significativa ( $p<0,05)$
\end{tabular}

25 días del ensayo. La tabla se encuentra organizada por promedios aritméticos y desviaciones estándar basados en la lectura final (25 días).

Como se puede ver en la Tabla 9, el tratamiento Vin Oz 30' (pH 7,4) presentó la producción acumulada de metano más alta igual a 104,3 mL de $\mathrm{CH}_{4}$ g DQO L ${ }^{-1}$ similar a la producida por el control 3 (AGV, 114,3 ml g DQO L ${ }^{-1}$ ). El control 3 no contiene compuestos inhibidores y la producción de metano es el resultado de la digestión del ácido acético, el ácido propiónico y el ácido butírico en proporción de 73:23:4 que sirvió como sustrato para el proceso de digestión anaerobia.

Los demás tratamientos tuvieron una producción de metano entre 23,3 a $45 \mathrm{ml}$ g DQO L-1. Se observa que los valores de producción de metano son más altos con vinazas pre-tratadas a $\mathrm{pH}$ cercanos a la neutralidad comparados con los obtenidos con vinazas pre-tratadas a pH ácidos; de la misma forma, la mayor producción de metano se obtuvo a 30 minutos de aplicación de ozono comparado con tiempos de 7 y 15 minutos. 
Tabla 12. Variación de la producción de metano frente al pH del sustrato

\begin{tabular}{c|c|c}
\hline pH & 4.4 & 7.4 \\
\hline $\begin{array}{c}\text { Producción de } \\
\text { metano (ml de } \\
\left.\mathbf{C H}_{4}\right)\end{array}$ & $16,11 \pm 21,8^{\mathrm{a}}$ & $60,82 \pm 36,53^{\mathrm{b}}$ \\
\hline \multicolumn{2}{|c}{ Media \pm desviación estándar $(\mathrm{n}=6)$} \\
Datos con diferentes letras en la misma fila indican diferencia \\
significativa ( $\mathrm{p}<0,05)$
\end{tabular}

De acuerdo al análisis de varianza de los resultados de la producción de metano para todos los tratamientos presentados en la Tabla 10, el aumento de la producción de metano para vinaza pre-tratada no depende de la interacción entre el tiempo de aplicación de ozono y el pH en los tratamientos, mientras que el tiempo de aplicación de ozono y pH intervienen individualmente sobre la producción de metano.

La Tabla 11 muestra la variación de la producción de metano para los diferentes tiempos de aplicación de ozono (7 - 15 - 30 minutos); como se puede observar, la producción de metano aumenta a medida que el tiempo de ozonización es más prolongado, demostrando la importancia de la aplicación de ozono como oxidante de compuestos de difícil degradación, favoreciendo el crecimiento de los microorganismos en el sistema anaerobio y consecuentemente la eficiencia en la producción de metano.

La Tabla 12 muestra la variación de la producción de metano frente al $\mathrm{pH}$, se observa que la mayor producción de metano se presenta a $\mathrm{pH}$ 7,4 lo que indica que la disminución de compuestos tóxicos en la vinaza se da en mayor proporción a un pH cercano a la neutralidad, esto puede ser atribuido a que la reacción de oxidación entre el ozono y el fenol está influenciada por el pH, siendo una relación directamente proporcional, es decir a medida que aumenta el pH aumenta la reacción de oxidación (Singer y Gurol, 1983).

\section{CONCLUSIONES}

El pre-tratamiento con ozono $\left(50 \mathrm{mg} \mathrm{L}^{-1} \mathrm{O}_{3}-\right.$ 30 min) demostró remoción de compuestos fenólicos en vinazas de caña de azúcar de $62 \%$ a pH 7,4 y de $35 \%$ a pH 4,4 .

La reacción de oxidación por ozono en una solución pura de fenol genera 89 \% de remoción de compuestos fenólicos. En las vinazas evaluadas es $27 \%$ y $54 \%$ más bajo el porcentaje de remoción a pH 7,4 y pH 4,4 respectivamente. Esta diferencia se debe principalmente a la naturaleza de las mezclas de compuestos fenólicos en el sustrato y al elevado contenido de materia orgánica que pueden actuar como inhibidores en el proceso de oxidación de los compuestos fenólicos (Forero et al, 2005).

La mayor producción de metano (104,3 ml g DQO $\mathrm{L}^{-1}$ ) se obtuvo de vinazas tratadas con ozono a $50 \mathrm{mg} \mathrm{L}^{-1}$ a 30 minutos de aplicación a pH 7,4 durante 25 días.

El tratamiento PAO $\left(\mathrm{O}_{3}\right)$ representa una buena opción para el mejoramiento de la producción de biogás a partir de residuos agroindustriales, ya que se demostró un aumento del $70 \%$ en la producción de metano a partir de vinazas pre-tratadas comparado con la producción de gas a partir de vinazas crudas.

\section{AGRADECIMIENTOS}

Los autores expresan su agradecimiento al grupo de investigación GAOX y la Universidad del Valle por la financiación y facilitar la realización de este estudio.

\section{REFERENCIAS}

Acevedo, D. M. (2011) Evaluación de tecnologías para la valoración de residuos orgánicos en la producción de AGV. (Tesis de maestría). Universidad del Valle; Santiago de Cali, Colombia.

APHA, AWWA y WEF. (2005) Standard Methods for the Examination of Water and Wastewater. (21th ed.) Washington D.C. 1368 p. 
Asocaña. (2011) Sector azucarero colombiano: informe anual de mercados. Área Económica. Santiago de Cali, Colombia. Asocaña.

- (2010) Sector azucarero colombiano: informe anual de mercados. Área Económica. Santiago de Cali, Colombia: Asocaña.

Caicedo, N. (2010) Pre-tratamiento con ozono de vinazas crudas provenientes de la industria de caña de azúcar. (Tesis de grado). Universidad del Valle: Santiago de Cali, Colombia.

Chernicharo, C. A. (2007) Principios do Tratamento Biológico de Águas Residuárias. Reatores anaeróbios. Universidade Federal de Minas Gerais, Departamento de Engenharia Sanitária e Ambiental. Brasil.

Cirne, D. G. et al. (2008). Control of Sulphide During Anaerobic Treatment of S-Containing Wastewaters by Adding Limited Amounts of Oxygen or Nitrate. Reviews in Environmental Science and Bio/ Technology, 7(2), June, pp. 93-105.

Dávila-Rincón, J.; Machuca-Martínez, F. y MarriagaCabrales, N. (2009). Reducción de demanda química de oxígeno, carbono orgánico total y sólidos totales en vinazas mediante electroflotación/oxidación. Revista Ingeniería e Investigación, 29(1), pp. 35-38.

España-Gamboa, E. I., et al. (2012). Methane Production by Treating Vinasses from Hydrous Ethanol Using a Modified UASB Reactor. Biotechnol Biofuels, 5(1), pp. 82-90.

España-Gamboa, E., et al. (2011). Vinasses: Characterization and Treatments. Waste Management \& Research, 29(12), pp. 1235-1250.

Federación Nacional de Biocombustibles de Colombia, (2015). Ventas de etanol en Colombia 2014-2015. Boletín No 135-Agosto 2015.

Forero, J.E.; Ortiz-Cancino, O.P. y Ríos, F. (2005). Aplicación de procesos de oxidación avanzada como tratamiento de fenol en aguas residuales industriales de refinería. Red de Revistas Científicas de América Latina y el Caribe, España y Portugal CT\&F Ciencia, Tecnología y Futuro. 3(1), enero, pp. 97-109.

García-Montaño, J.; et al. (2008). The Testing of Several Biological and Chemical Coupled Treatments for
Cibacron Red FN-R azo dye removal. Journal of Hazardous Materials, 154(1-3), pp. 484-490.

Gil, J.J. (2011) Evaluación de la producción de metano en la digestión anaerobia de vinazas pretratadas con un proceso avanzado de oxidación. (Tesis de maestría). Universidad del Valle: Santiago de Cali, Colombia.

Gogate, P y Pandit, A. (2004). A Review of Imperative Technologies for Wastewater Treatment I: Oxidation Technologies at Ambient Conditions. Advances in Environmental Research, 8(3-4), March, pp. 501551.

Global Renewable Fuels Alliance, (2014). Global Ethanol Production Will Rise to Over 90 Billion Litres in 2014. [en línea] Toronto, Canada. Disponible en: http://globalrfa.org/news-media/global-ethanolproduction-will-rise-to-over-90-billion-litresin-2014.

Jeworski, M. y Heinzle, E. (2000). Combined Chemical Biological Treatment of Wastewater Containing Refractory Pollutants. Biotechnology Annual Review, 6, pp. 163-196.

Lorenzo-Acosta, Y. y Obaya-Abreu, M. C. (2005). La digestión anaerobia. Aspectos teóricos. Parte I. ICIDCA. Sobre los Derivados de la Caña de Azúcar, 39 , pp. 35-48.

Malato, S.; et al. (2009). Decontamination and Disinfection of Water by Solar Photocatalysis: Recent Overview and Trends. Catalysis Today, 147(1), pp. 1-59.

Martín-Santos, Ma.; et al. (2003). Ozonation of Vinasse in Acid and Alkaline Media. Journal of Chemical Technology and Biotechnology, 78(11), November, pp. 1121-1127.

Martín-Santos, Ma.; et al. (2005). Estimating the Selectivity of Ozone in the Removal of Polyphenols From Vinasse. Chemical Technology, 438(4), April, pp. 433-438.

Parawira, W.; et al. (2008). Energy Production from Agricultural Residues: High Methane Yields in PilotScale Two-Stage Anaerobic Digestion. Biomass and Bioenergy, 32(1), January, pp. 44-50.

Renewable Fuels Association. (2011) World Ethanol Production Outlook. [en línea] Renewable Fuels Association. Disponible en http://www.ethanolrfa. org/pages/statistics 
Riberio, K. (2007) Avaliação Técnico-Econômica e Ambiental da Utilização do Biogás Proveniente da Biodigestão da Vinhaça em Tecnologias para Geração de Eletricidade. (Tesis doctoral) Universidade Federal de Itajubá: Itajubá, Brasil.

Robles-González, V.; et al. (2012). Treatment of Mezcal Vinasses: a Review. Journal of biotechnology, 157(4), February, pp. 524-546.

Roesler, R.; et al. (2007). Atividade Antioxidante de Frutas do Cerrado. Revista Ciência e Tecnología de Alimentos, Campinas. 27(1), pp. 53-60.

Siles, J.A.; et al. (2011). Integrated Ozonation and Biomethanization Treatments of Vinasse Derived from Etanol Manufacturing. Journal of Hazardous Materials, 188(11), pp. 247-253.

Singer, P.C. y Gurol, M.D. (1983). Dynamics of the Ozonation of Phenol. Experimental Observations. Water Research, 17(9), pp. 1163-1171.

Travaini, R.; et al. (2013). Sugarcane Bagasse Ozonolysis Pretreatment: Effect on Enzymatic Digestibility and Inhibitory Compound Formation. Bioresource technology, 133, April, pp. 332-339.

Yusuf, Y. (2007). EC and EF Processes for the Treatment of Alcohol Distillery Wastewater. Separation and purification technology, 53, pp. 135-140.

\section{PARA CITAR ESTE ARTÍCULO / TO REFERENCE THIS ARTICLE / PARA CITAR ESTE ARTIGO /}

Durán-Meneses, M.F.; Sanabria-Gómez, I.J.; GutiérrezGuzmán, N.(2015). Evaluación de la producción de metano en la digestión anaerobia de vinazas pretratadas con ozono. Revista EIA, 12(24), Julio-diciembre, pp. 167-177. [Online]. Disponible en: DOI: http:/dx.doi.org/10.14508/ reia.2015.12.24.167-177 